\title{
Liner impedance eduction under shear grazing flow at a high sound pressure level
}

\author{
F. Méry*, V. Lafont ${ }^{\dagger}$, R. Roncen ${ }^{\ddagger}$, F. Simon ${ }^{\S}$ and E. Piot ${ }^{\Uparrow}$ \\ ONERA/DMPE - Université de Toulouse, F-31055, Toulouse, France
}

\begin{abstract}
This paper investigates the combined effects of high sound pressure level and grazing flow on impedance eduction for classical liners. Experiments are conducted in the grazing flow duct at ONERA (B2A). The impedance is then educed with an inverse method adapted to a shear flow. To take into account the effects of incident sound pressure level, a new strategy for impedance eduction is developed, using a space-dependent variable term. The new strategy is applied to different experimental cases and the results are compared to those obtained with the classical method.
\end{abstract}

\section{Nomenclature}

$\begin{array}{ll}\phi & \text { Liner perforated sheet perforation diameter, }(\mathrm{mm}) \\ \delta & \text { Liner perforated sheet thickness, }(\mathrm{mm}) \\ L_{\text {total }} & \text { Liner sample length }(\mathrm{m}) \\ \rho_{0} & \text { Density of the mean flow, }\left(\mathrm{kg} / \mathrm{m}^{3}\right) \\ M_{b} & \text { Bulk flow Mach number } \\ \omega & \text { Pulsation, }(\mathrm{rad} / \mathrm{s}) \\ Z & \text { Normalized acoustic impedance } \\ R & \text { Resistance, real part of } Z \\ X & \text { Reactance, imaginary part of } Z \\ \beta & \text { Acoustic reflection coefficient at normal incidence } \\ Z_{t} & \text { Normalized exit impedance } \\ \mathcal{C} & \text { Source amplitude (complex coefficient) } \\ (x, y, z) & \text { Axial, transversal and vertical coordinates, }(\mathrm{mm}) \\ (U, W) & \text { Axial and vertical mean velocity, }(\mathrm{m} / \mathrm{s}) \\ (u, w) & \text { Axial and vertical acoustic velocity, }(\mathrm{m} / \mathrm{s}) \\ c_{0} & \text { Sound celerity, (m/s) } \\ R_{L} & \text { Resistance, constant part } \\ R_{N L}(x) & \text { Resistance, variable part } \\ L_{\tau} & \text { Characteristic length of SPL effects on } Z,(\mathrm{~m})\end{array}$

\section{Introduction}

The effect of a complex flow on the acoustical impedance of nacelle liners remains a topical and challenging subject for nacelle liner design. Eduction methods are now a widely spread technique used to retrieve the acoustic impedance of liner materials with indirect non-intrusive measurements, in the presence of a grazing flow. Some methods are straightforward, generally using a measurement of an axial propagation constant related to the impedance condition $1{ }^{12}$ Indirect methods usually consist in measuring the acoustic pressure

\footnotetext{
*Research Scientist, fabien.mery@onera.fr

$\dagger$ PhD Student, victor.lafont@onera.fr

${ }^{\ddagger}$ Research scientist, remi.roncen@onera.fr

$\S$ Research scientist, frank.simon@onera.fr

๑Research scientist, estelle.piot@onera.fr
} 
on the wall opposite the liner and using a minimization method where experimental and numerical results are compared. In order to describe more precisely the local effects on the liner, ONERA applied a Laser Doppler Anemometry (LDA) technique to measure the acoustic velocity fields in the area above the liner, and used them as inputs for the eduction process. ${ }^{3 / 5}$ The numerical resolution of the Linearized Euler Equations (LEE) was obtained with a discontinuous Galerkin scheme, accounting naturally for the presence of a shear flow in the simulation. The main challenge is to ensure the best reliability of the eduction process and high accuracy of the results. ${ }^{6}$ Recently, Roncen et al ${ }^{7}$ dealt with a new approach based on Bayesian inference in order to have an evaluation of the uncertainties on the impedance results. For a classical perforate-overhoneycomb liner, it is usually assumed that the liner impedance is constant along the streamwise direction. However, when the incident Sound Pressure Level (SPL) is high, a phenomenon of vortex shedding appears in the vicinity of the perforations. $\stackrel{8}{ }$ This leads to a modification of the impedance (mainly an increase of the resistance) 9 [11 These studies show that the impedance depends on the incident sound pressure level in the presence of high SPL. In a lined duct configuration, the sound pressure level may be much higher upstream of the liner than downstream of the liner. It seems thus relevant to take into account the variation of the impedance as a function of the variation of the incident sound pressure level which decreases along the liner. Jones ${ }^{12}$ recently educed the impedance of multisegment liners, where the studied liners had a piecewise impedance evolution along the streamwise direction.

The objective of this paper is to apply the same kind of eduction strategy on a classical Single Degree of Freedom (SDOF) liner to take into account the non-linear effects due to the incident sound pressure level with a shear grazing flow. Section II is dedicated to the presentation of the IFAR liner sample used for this study and the experimental set up, while Section III proposes a general presentation of ONERA eduction methods and its application on the IFAR liner. Section IV emphasizes the modifications on the eduction process in order to take into account the SPL effect.

\section{Description of the experimental setup}

\section{A. Liner definition}

Single degree of freedom liners are typically made using a honeycomb structure that is backed by a rigid plate and topped by a perforated facesheet, as seen on Fig. 1.

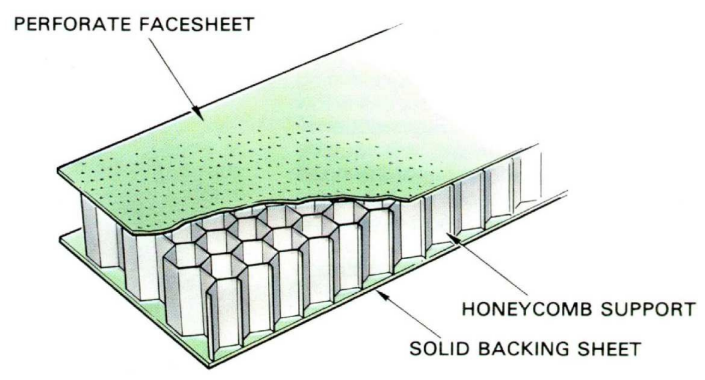

Figure 1. Sketch of a conventional SDOF liner

In this study, two of these classical SDOF liners are used. The first sample was designed in the framework of the IFAR benchmark challenge \#1 and manufactured by an additive process. It has square core chambers, an integrated conventional facesheet, and a core depth of $50.8 \mathrm{~mm}$. The second liner was manufactured for a previous study and already tested by NASA $\stackrel{13}{13}$ Its chambers are hexagonal and it is less thick than the IFAR sample. Table 1 sums up the characteristics of both samples.

Different empirical models exist to describe the behavior of liners at high sound pressure level or high flow speeds,$\frac{14}{14}$ depending on their characteristics. Based on these models, both liner samples should exhibit non-linear effects due to incident SPL at around the same acoustic level. 
Table 1. Characteristics of the liner samples

\begin{tabular}{lcc}
\hline \hline & IFAR sample & ONERA sample \\
\hline Facesheet thickness $(\delta)$ & $0.86 \mathrm{~mm}$ & $0.8 \mathrm{~mm}$ \\
Hole diameter $(\phi)$ & $1.04 \mathrm{~mm}$ & $0.3 \mathrm{~mm}$ \\
Percent Open Area (POA) & $11 \%$ & $5 \%$ \\
Core cell size & $10.16 \mathrm{~mm} \times 10.16 \mathrm{~mm}$ & $9.5 \mathrm{~mm}$ (diameter) \\
Partition thickness & $2.54 \mathrm{~mm}$ & $0.1 \mathrm{~mm}$ \\
Core depth & $50 \mathrm{~mm}$ & $20 \mathrm{~mm}$ \\
\hline \hline
\end{tabular}

\section{B. Measurement}

\section{ONERA B2A duct}

The aeroacoustic test bench at ONERA was developed and built in 2004 $\sqrt{15}[16$ It is made of a stainless steel tube of section $50 \mathrm{~mm} \times 50 \mathrm{~mm}$ and has a total length of about $4 \mathrm{~m}$. A $0.2 \mathrm{~m}$-long test section is equipped with two silica windows for optical access. The termination is equipped with a quasi-anechoic outlet, which leads to a reflection coefficient smaller than 0.2 for frequencies higher than $500 \mathrm{~Hz}$. A mean flow of bulk Mach number $M_{b}$ up to 0.5 can be provided. The flow temperature can be accurately regulated, from the ambient temperature up to $570 \mathrm{~K}$. All along the study, the flow temperature was imposed to be equal to the ambient temperature $(290 \mathrm{~K})$. In the test section, this flow shows fully developed turbulent boundary layers, with a turbulence rate of a few percent at the center of the section. In Fig. 2, flow propagates from left to right.

Two acoustic drivers are mounted upstream of the test section, and are used to generate tones (usually a multi-sine signal) at up to $150 \mathrm{~dB}$ over a frequency range of 0.3 to $3.5 \mathrm{kHz}$ (i.e., the no-flow cut-off frequency of the duct for plane waves). When a multi-sine source is used, the overall SPL (OASPL) within the duct is much higher (usually $10 \mathrm{~dB}$ more) than the SPL at each tone frequency. The surface of the test liner forms a $150 \mathrm{~mm}$-long portion of the lower wall of the flow duct and spans all the duct width.

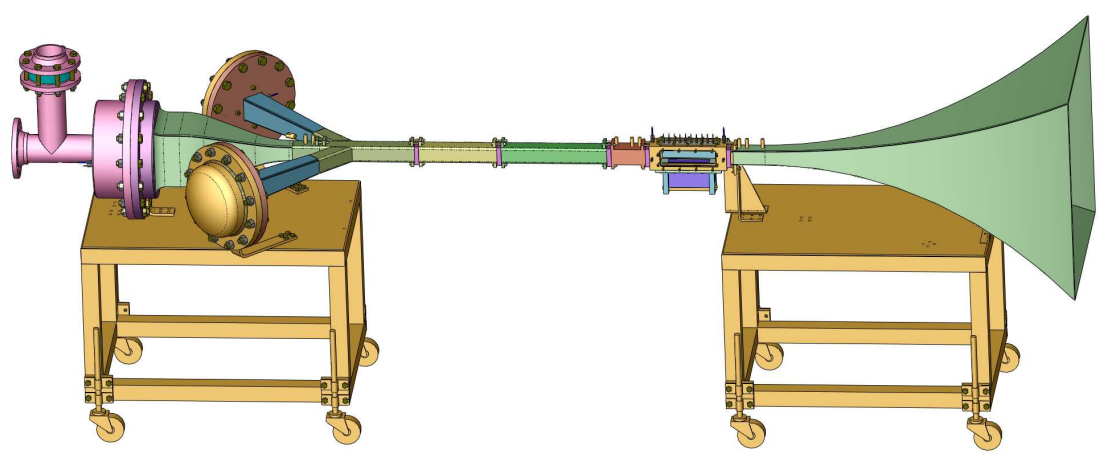

Figure 2. Sketch of B2A Aero-Thermo-Acoustic test bench.

Flush-mounted GRAS 40SA microphone probes are used (probe diameter of $1.2 \mathrm{~mm}$ ). Usually, two upstream locations are considered to measure the amplitude of the incident acoustic plane waves ${ }^{17}$ However, for the pressure-based impedance eduction method, one microphone probe is moved along the whole set of microphone locations. The cross-spectrum between the microphone (or the LDA system) and driver signals is acquired, which provides a phase reference for the pressure measurements.

\section{Two-microphone method for measuring the incident and reflected pressure waves}

A two-microphone method is used to decompose the measured sound field in the duct into an incident and a reflected wave ${ }^{[17}$ This analysis is only performed below the first cut-off frequency of the duct, i.e. under $3.5 \mathrm{kHz}$, so that only plane waves are considered. This two-microphone method is used to assess the incident 
SPL onto the sample, i.e the SPL of the downstream wave denoted by $A$ in Fig. 3 , while the upstream reflected wave is denoted by $B$.

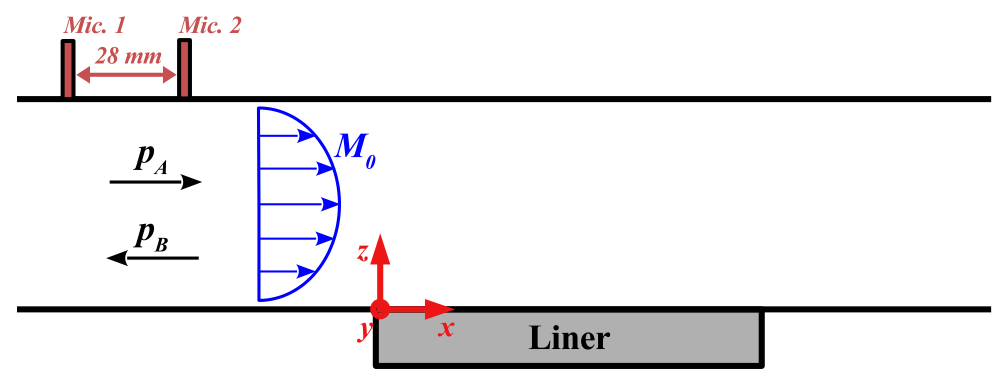

Figure 3. Sketch of the wave sorting procedure.

Two microphone probes GRAS 40SA are placed flush to the wall, at a distance $x_{1}$ and $x_{2}$ of the sample and therefore of the wall impedance discontinuity. We note $p_{1}$ and $p_{2}$ the sound pressure they measure. The spacing between the two probes is $28 \mathrm{~mm}$, which according to Boden \& Abom $\frac{18}{18}$ minimizes errors in the $[600,3450] \mathrm{Hz}$ frequency range. In order to also minimize measurement errors at lower frequencies, the measurements are repeated by switching the two microphones and an average of the obtained frequency response function is taken: this makes it possible to overcome the intrinsic phase shift between the microphones. Using the complex notation, and by introducing the angular frequency $\omega=2 \pi f$, the pressure field in the test section at a given position $x$ reads

$$
p(x, t)=\operatorname{Re}\left(P(x) e^{\mathrm{j} \omega t}\right),
$$

with $P(x)=A e^{-\mathrm{j} k^{+} x}+B e^{\mathrm{j} k^{-} x}$ where $k^{+}$and $k^{-}$are the wave numbers, $A$ and $B$ are the complex amplitudes of the forward and backward waves, and $\mathrm{j}$ is the complex unit. The $x$-axis is oriented from left to right and has its origin at the liner upstream location, i.e. at the position of the impedance discontinuity. For a uniform flow of Mach number $M$, we have:

$$
k^{ \pm}= \pm \frac{\omega / c_{0}}{1 \pm M}=\frac{ \pm k}{1 \pm M}
$$

with $k=\omega / c_{0}$.

The two-microphone method allows the assessment of the sound pressure level $S_{A}$ of the downstream wave (i.e. $20 \log \frac{P_{A}^{r m s}}{2.10^{-5}}$ with $P_{A}^{r m s}=\frac{|A|}{\sqrt{2}}$ ) from the sound pressure levels $S_{1}$ and $S_{2}$ measured at the microphones, by solving the following set of equations:

$$
\begin{aligned}
& A e^{-\mathrm{j} \frac{k}{1+M} x}+B e^{\mathrm{j} \frac{k}{1-M} x}=P_{1}, \\
& A e^{-\mathrm{j} \frac{k}{1+M} x}+B e^{\mathrm{j} \frac{k}{1-M} x}=P_{2},
\end{aligned}
$$

which yields the following expressions for the SPL of the downstream and upstream waves (in decibels, $S_{B}$ being defined from $|B|$ in the same way as $S_{A}$ has been defined from $|A|$ ):

$$
\begin{aligned}
& S_{A}=S_{1}-20 \log \left(2\left|\sin \frac{k \Delta x}{1-M^{2}}\right|\right)+10 \log \left(1+2\left|H_{21}\right| \cos \left(\phi_{21}-\frac{k \Delta x}{1-M}\right)+\left|H_{21}\right|^{2}\right) \\
& S_{B}=S_{1}-20 \log \left(2\left|\sin \frac{k \Delta x}{1-M^{2}}\right|\right)+10 \log \left(1+2\left|H_{21}\right| \cos \left(\phi_{21}-\frac{k \Delta x}{1+M}\right)+\left|H_{21}\right|^{2}\right)
\end{aligned}
$$

with $\Delta x=x_{2}-x_{1}$ and $H_{21}=\left|H_{21}\right| e^{i \phi_{21}}=P_{2} / P_{1}$, the frequency response function between the microphones. More details on the resolution process can be found in a previous paper.19] In the present study, $M$ is chosen equal to $M_{b}$, the bulk flow Mach number. 


\section{Non-intrusive measurement of the acoustic velocity field}

The B2A duct is equipped with a LDA system, in order to measure the streamwise and vertical velocity components $u$ and $w$ of the flow. As this measurement technique is time-resolved, both the mean and fluctuating parts of the velocity can be measured. Especially, the acoustic velocity field can be obtained with a specific post-processing technique ${ }^{20}$ The acoustic velocity is defined here as the component of the signal that is correlated with the loudspeaker signal. It can be educed from the extraneous noise (mainly due to the turbulent flow) by a technique similar to the three-microphone signal enhancement technique $\frac{17 \sqrt{21}}{16}$ It consists in calculating the cross-spectral density function $G_{U_{i}, l s}$ between the velocity signal $U_{i}$ and the

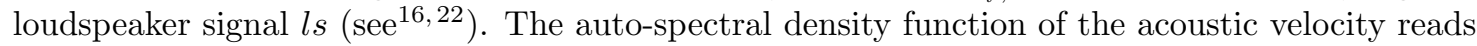

$$
G_{u_{i}}=\frac{\left|G_{U_{i}, l s}\right|^{2}}{G_{l s}}
$$

where $G_{l s}$ is the auto-spectral density function of the loudspeaker signal. The acoustic velocity in the frequency domain is then given by

$$
u_{i}=\sqrt{G_{u_{i}}} \exp \left[\mathrm{j} \Phi\left(U_{i} / l s\right)\right]
$$

where the phase of the acoustic velocity, referenced by the loudspeaker, is defined as

$$
\Phi\left(U_{i} / l s\right)=\arctan \frac{\operatorname{Im}\left(G_{U_{i}, l s}\right)}{\operatorname{Re}\left(G_{U_{i}, l s}\right)} .
$$

The uncertainty in the measurement of the velocity field is estimated with a Bootstrap approach which allows to evaluate the variance associated to the amplitude and phase of the measured field.7

\section{4. $\quad$ Test Matrix}

The different tested configurations for the IFAR sample are presented in Table 2 The acoustic excitation consists of 12 pure tones distributed between $504 \mathrm{~Hz}$ and $2824 \mathrm{~Hz}$, each having the same acoustic level. The multi-sine excitation is set in order to have the same incident sound pressure level for each tone. The $120 \mathrm{~dB}$ multi-sine excitation has thus an incident OASPL of $130 \mathrm{~dB}$, and the $130 \mathrm{~dB}$ multi-sine excitation has an OASPL of $140 \mathrm{~dB}$. The selected frequency for mono sine excitations is close to the resonance of the sample (i.e. close to the maximum of absorption): $f=1528 \mathrm{~Hz}$.

The test matrix for the ONERA sample is similar to the test matrix for the IFAR sample. This time, the excitation is composed of 10 pure tones, distributed between $992 \mathrm{~Hz}$ and $3136 \mathrm{~Hz}$. The resonance of the liner occurs around $f=1992 \mathrm{~Hz}$, this frequency is thus chosen for the single tone excitation (case \#8).

Table 2. Test matrix

\begin{tabular}{cccccc}
\hline \hline \# Case & Sample & SPL $[\mathrm{dB}]$ on each tone & OASPL $[\mathrm{dB}]$ & $M_{b}$ & Excitation type \\
\hline 1 & IFAR & 120 & 130 & 0.1 & Multi-sine \\
2 & IFAR & 130 & 140 & 0.1 & Multi-sine \\
3 & IFAR & 140 & 140 & 0.1 & Single tone \\
4 & IFAR & 150 & 150 & 0.1 & Single tone \\
5 & IFAR & 130 & 140 & 0.3 & Multi-sine \\
6 & ONERA & 120 & 130 & 0.2 & Multi-sine \\
7 & ONERA & 130 & 140 & 0.2 & Multi-sine \\
8 & ONERA & 130 & 130 & 0.2 & Single tone \\
\hline \hline
\end{tabular}

\section{Impedance eduction approach}

\section{A. Formulation}

In the B2A configuration, an impedance eduction method based on the two-dimensional time-harmonic linearized Euler equations is used. The underlying model is briefly summarized here for convenience, but all details can be found elsewhere $\frac{5}{5}$ 
The LEE, written in non-conservative form, with an $e^{\mathrm{j} \omega t}$ time dependence, assuming homentropic flow is

$$
\mathbf{L} \varphi=0
$$

where

and

$$
\mathbf{L} \boldsymbol{\varphi}=\mathrm{j} \omega \boldsymbol{\varphi}+\boldsymbol{A}_{x} \frac{\partial \boldsymbol{\varphi}}{\partial x}+\boldsymbol{A}_{z} \frac{\partial \boldsymbol{\varphi}}{\partial z}+\boldsymbol{B} \boldsymbol{\varphi}
$$

$$
\boldsymbol{A}_{x}=\left(\begin{array}{ccc}
U & 0 & c_{0} \\
0 & U & 0 \\
c_{0} & 0 & U
\end{array}\right), \boldsymbol{A}_{z}=\left(\begin{array}{ccc}
W & 0 & 0 \\
0 & W & c_{0} \\
0 & c_{0} & W
\end{array}\right), \boldsymbol{B}=\left(\begin{array}{ccc}
\partial_{x} U & \partial_{z} U & 0 \\
\partial_{x} W & -\partial_{x} U & 0 \\
0 & 0 & 0
\end{array}\right)
$$

Components of vector $\varphi=\left(u, w, \frac{p}{\rho_{0} c_{0}}\right)$, where $\rho_{0}$ is the density of the mean flow, represent the acoustic perturbations around the sheared mean flow of axial and transverse velocities $U$ and $W$, respectively. The flow being assumed homentropic, the energy equation is replaced by the state equation $p=c_{0}^{2} \rho_{0}$.

As we consider a shear mean flow profile, the impedance boundary condition on the lined wall is the standard one

$$
p= \pm \rho_{0} c_{0} Z w,
$$

where the sign is chosen depending on whether the upper or lower wall is considered. $Z$ is first considered as constant all along the lined zone. To avoid any singularities in the rigid wall case, the boundary condition (12) is expressed as a function of the normal incidence reflection coefficient $\beta=(Z-1) /(Z+1)$.

At the source plane, the state vector $\boldsymbol{\varphi}$ is taken to be equal to $\mathcal{C} \boldsymbol{\varphi}_{0}$, where $\boldsymbol{\varphi}_{0}$ is the plane-wave solution in a rigid duct with uniform flow Mach number $M_{0}$, corresponding to the bulk Mach number of the actual shear flow $U(z)$. As this plane-wave solution is chosen with an arbitrary amplitude taken to be equal to 1 , the complex coefficient $\mathcal{C}$ stands for the source amplitude. $\mathcal{C}$ can be either set to the measured value of the acoustic pressure at the source plane, when available, or included into the eduction process to reproduce at best the measured acoustic field. At the exit plane, either a non-reflecting or an exit impedance boundary condition can be enforced. The first option is only used when the data suggests that this is proper, i.e. when no standing-wave can be observed in the downstream hard wall section. As the LDA measurement system does not extend to the exit plane, this information can only be provided by microphone measurements downstream of the lined section. As these additional measurements are not systematically performed, the exit impedance boundary condition is commonly used. This will be the case for all the computations shown in this paper. As a consequence, during the search procedure, both $\mathcal{C}$ and $Z_{t}$ are used as design variables, in addition to $Z$.

A discontinuous Galerkin (DG) scheme is chosen to solve Eq. (10) and the associated boundary conditions. In this method, discontinuities are allowed at the interface between two elements. An example of how such a method can handle discontinuities at hard-soft wall interfaces is found elsewhere. ${ }^{23}$ A triangular mesh of Lagrange $\mathbb{P}_{1}$ elements is used. To ensure the connection between elements and to apply the boundary conditions, a numerical flux is defined. An upwind numerical flux is used to ensure the connection between interior cells, while a centered flux is chosen at the boundaries (see previous paper 5524 for more details).

The inverse problem is defined by the minimization of the following objective function:

$$
\mathcal{J}_{\text {red }}=\frac{\int_{\Omega_{o b s}}\left\{\left.\boldsymbol{\varphi}\right|_{\mathrm{DG}}-\left.\boldsymbol{\varphi}\right|_{\text {Meas }}\right\}^{\top}\left\{\left.\overline{\boldsymbol{\varphi}}\right|_{\mathrm{DG}}-\left.\overline{\boldsymbol{\varphi}}\right|_{\text {Meas }}\right\} \mathrm{d} x \mathrm{~d} z}{\int_{\Omega_{\text {obs }}}\left\|\left.\boldsymbol{\varphi}\right|_{\text {Meas }}\right\|^{2} \mathrm{~d} x \mathrm{~d} z},
$$

where $\Omega_{o b s}$ is the observation region.

For this study, the impedance eduction method is based on LDA measurements above the test liner (see Fig. 4), $\Omega_{o b s}$ is a rectangular $(x, z)$ region and the $(u, w)$ velocity components of the state vector are considered in function (13).

In this study, the optimization problem is solved using a CMAES method, with the help of a surrogate model. A statistical inference method developed by Roncen et al. in 2018 can be also applied to compute uncertainties on the educed values. ${ }^{7}$ This method is based on a Bayesian approach, and uses a MCMC (Markov Chain Monte Carlo) algorithm instead of the CMAES technique. Coupled with the bootstrap method for estimating the errors on LDA measurements, it gives a good appreciation of the confidence intervals on the educed impedance. 


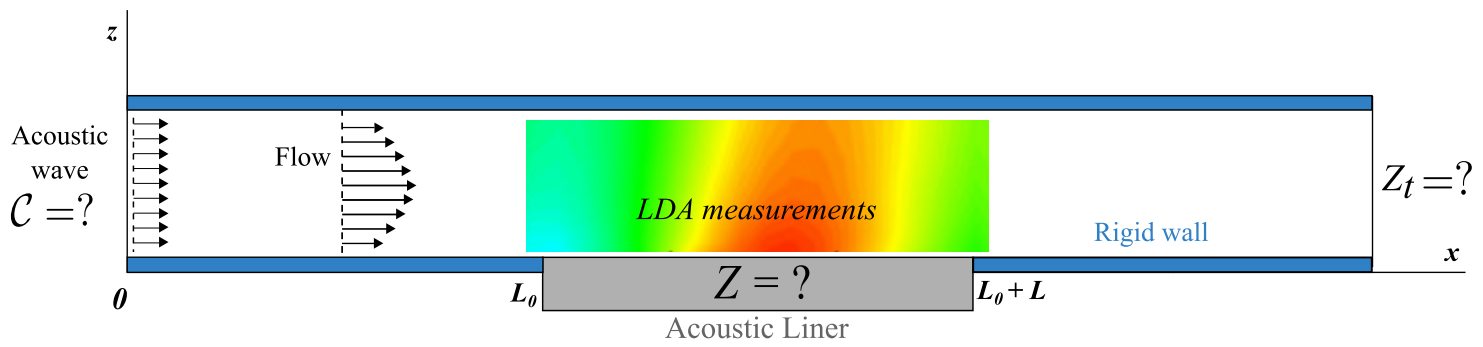

Figure 4. Impedance eduction from LDA measurements

\section{B. Classical impedance eduction results}

This section presents the results of the CMAES algorithm on the five cases presented in Table 2 The goal is to study the behavior of the educed impedance with respect to the bulk Mach number and the incident SPL.

Figure 5 highlights the effect of the Mach number on the impedance. The main effects are on the resistance which increases with the Mach number. The reactance is almost the same and on this liner configuration, no effect is noticeable on the reactance term, which is a known classical result.

Figure 6 is dedicated to show the SPL effect on the impedance, at a fixed grazing flow speed. One can notice an increase of the resistance when the OASPL is increased at the frequency where the absorption is maximum, i.e. near the liner resonance $(X=0)$.

In the case of mono-sine excitation, the incident SPL and the OASPL are the same as there is only one tone. Thus, the OASPL for an incident SPL of $140 \mathrm{~dB}$ and a mono-sine excitation is very close to the OASPL for a multi-sine excitation with $130 \mathrm{~dB}$ on each tone. For these two cases, the educed resistance values shown on Fig. 6 are very comparable: 0.307 and 0.345 for case \#3 and \#4 respectively. For an incident SPL of $150 \mathrm{~dB}$, the effect is greater: the resistance increases to 0.52 around the liner resonance. However, the reactance is unresponsive to the sound pressure effect and remains the same for the considered frequency.

The incident OASPL is the driving parameter to exhibit the effect of the sound pressure level on the resistance. In the following sections, we will focus on the monosine excitation results since this excitation enables to reach higher levels (up to $150 \mathrm{~dB}$ ), so as to ensure a maximum effect on the impedance.

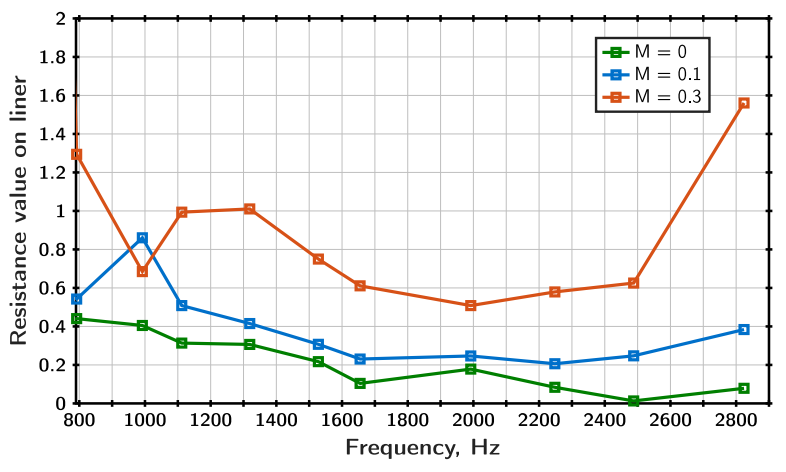

(a)

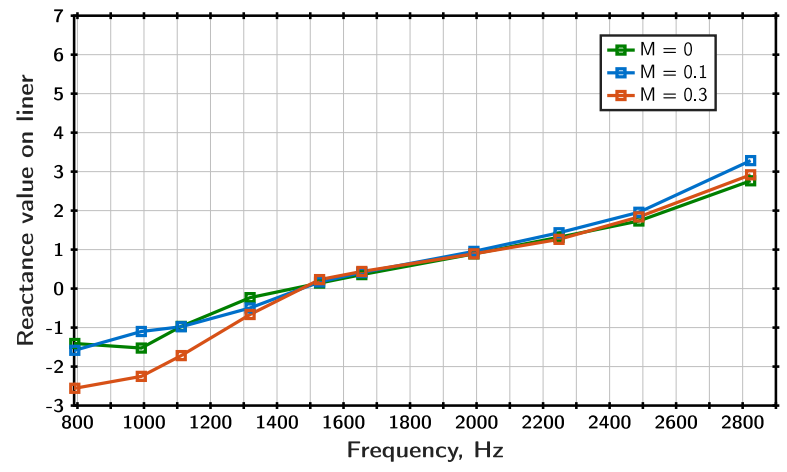

(b)

Figure 5. Effect of the bulk Mach number on the eduction result for multi-sine source with $130 \mathrm{~dB}$ incident SPL on each tone, (a) Normalized resistance and (b) Normalized reactance 


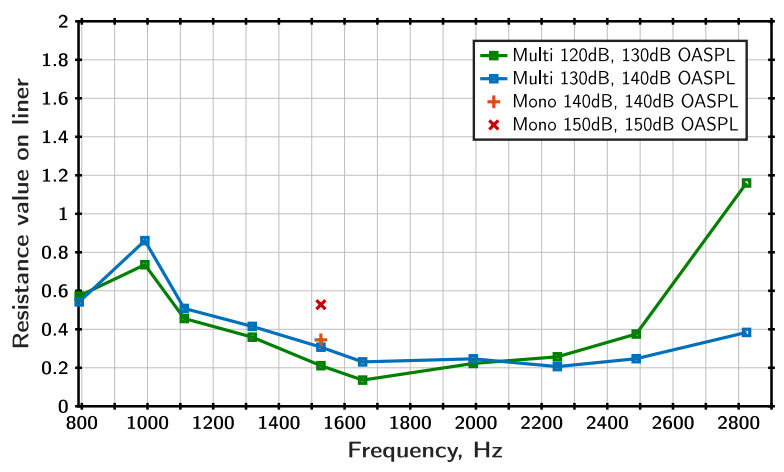

(a)

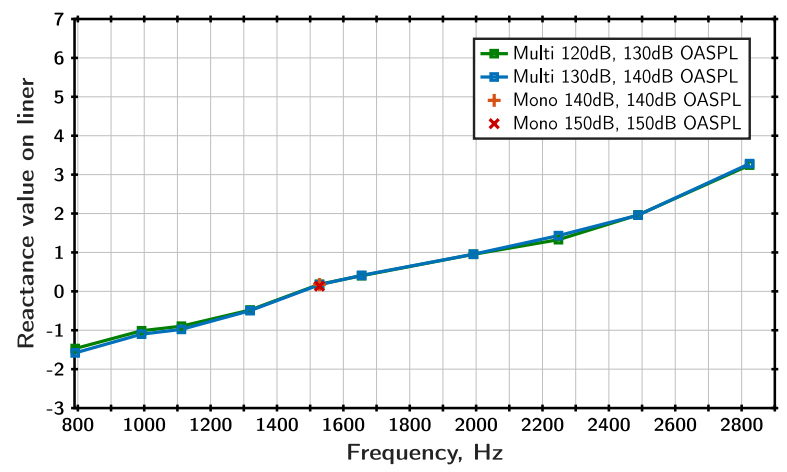

(b)

Figure 6. Effect of Sound Pressure Level on the eduction result for $M_{b}=0.1$, (a) Normalized resistance and (b) Normalized reactance

\section{Taking into account non-linear behavior in the eduction process}

\section{A. Streamwise varying liner impedance boundary condition}

In the standard eduction process, the impedance boundary condition on the lined wall is constant all along the liner. High sound pressure level implies the creation of vortices and their detachment is done alternately on both sides of the perforation over two half-periods of the acoustic excitation. The discharge coefficient is defined by the ratio between the core inflow-to-outflow area on each orifice of the perforated plate. The inflow area is reduced due to the apparition of a "vena contracta" due to the vortex shedding. This effect was first exhibited by Hersh $\stackrel{8}{\text { Cummings }} \frac{11}{11}$ proposed a calculation of this discharge coefficient $C_{D}$. The effect of the sound pressure level on the liner resistance is presented as an additive term related to a constant coefficient and the normal acoustic velocity $|v|$ in each hole.

The model on the resistance term can be written as:

$$
R=R_{L}+C|v|
$$

where $R_{L}$ is the value of the resistance in the linear regime, $C$ is a correction coefficient related to the discharge coefficient $C_{D}$ and $|v|$ is the normal acoustic velocity. This last component is directly linked to the sound pressure level. Cummings proposed a way of calculating this discharge coefficient. Tonon et al. proposed other formulations for the calculation of the discharge coefficient. 25 Zhang \& Bodony ${ }^{29}$ performed DNS computation and confirmed that the discharge coefficient is directly linked to the sound pressure level. Very recently, Rienstra \& Singh 10 managed to analytically calculate this non-linear effect and showed that this effect is even more important at the resonance of the liner. In the rest of the article, we will focus on excitation frequencies close to the resonance. The challenge here is to catch the impedance variation due to high sound pressure level with our eduction procedure with shear grazing flow. The idea is to educe the non-linear part of the resistance while taking into account the decrease of the sound pressure level along the lined region. In the stream-wise direction, the impedance of the liner is no more constant and reads

$$
Z(x)=R_{L}+j X+R_{N L}(x),
$$

where $R_{N L}(x)$ is a decreasing function.

Different models are proposed for $R_{N L}(x)$. All of them include a length parameter $L_{\tau}$ which represents the length of the part of the liner over which the resistance is expected to change due to the SPL effect: when $x>L_{\tau}, R_{N L}(x)$ vanishes and only the constant term $R_{L}$ remains. Thus, the only difference between the models is the function $R_{N L}(x)$ : one simple approach is to use decreasing polynomial functions of degree 1 or higher, another possibility is the use of exponential functions. The three models retained for the study are shown in Table 3. For simplicity, the liner is considered to start at $x=0$ even if it not always exactly the case in the experimental setup. These three variable impedance boundary conditions are implemented to the LEE simulation and the educed parameters are modified accordingly. 
Table 3. Impedance boundary condition formulations

\begin{tabular}{lll}
\hline \hline \# Formulation & $Z(x)$ & Educed parameters \\
\hline (0) Constant & $R+j X$ & $\left(R, X, Z_{t}, \mathcal{C}\right)$ \\
(I) Affine & $R_{L}+j X+D\left(x-L_{\tau}\right) \times \mathbb{1}_{0<x<L_{\tau}}(x)$ & $\left(R_{L}, X, D, L_{\tau}, Z_{t}, \mathcal{C}\right)$ \\
(II) Polynomial & $R_{L}+j X+\left(D_{1}\left(x-L_{\tau}\right)+D_{2}\left(x-L_{\tau}\right)^{2}\right) \times \mathbb{1}_{0<x<L_{\tau}}(x)$ & $\left(R_{L}, X, D_{1}, D_{2}, L_{\tau}, Z_{t}, \mathcal{C}\right)$ \\
(III) Exponential & $R_{L}+j X+A \exp \left(-\frac{x}{L_{\tau}}\right)$ & $\left(R_{L}, X, A, L_{\tau}, Z_{t}, \mathcal{C}\right)$. \\
\hline \hline
\end{tabular}

\section{B. Eduction results with a variable impedance value}

In this section, the new eduction process is applied to the measurement on the IFAR liner sample.

\section{Comparison of the three variable conditions}

Figure 7 shows the results for the 3 new boundary formulations and the classical constant impedance for an incident SPL of $150 \mathrm{~dB}$, with $M_{b}=0.1$ and $f=1528 \mathrm{~Hz}$. The three impedance profiles are close in terms of evolution along the liner and starting value at $x=0$.

To emphasize the efficiency of these new boundary conditions, we rely on the cost function. In Table 4 (I), (II) and (III) liner impedance boundary conditions are compared for the case with highest OASPL, where the effects are expected to be the most important. Changing from a constant to a variable impedance is clearly efficient to minimize the cost functions as the new cost is 6 times smaller than the original cost, however the improvement between conditions (I), (II) and (III) is small. Due to the way the calculation has to be computed, using either (II) or (III) liner impedance boundary conditions implies more time-consuming calculations with a low benefit on the cost function minimization.

In Table 5, the affine model (I) is applied for several sound pressure excitations. It can be noticed that as the incident SPL becomes greater the use of the variable liner impedance boundary condition is efficient to minimize the cost function. On the contrary, when the incident SPL is low (as in case \#1), the gain is not significant. The evolution along the liner is thus indeed linked to SPL effects. This analysis of the cost functions shows that the affine liner impedance boundary condition (I) is sufficient. Moreover, it gives a very simple numerical formulation. In the following sections, we will therefore only focus on this boundary condition and compare it with the classical constant impedance.

Table 4. Comparison between (I), (II) and (III) formulations, OASPL = 150 dB, $f=1528 H_{z}, M_{b}=0.1$ (case \#4)

\begin{tabular}{lc}
\hline \hline Boundary formulation & Cost function \\
\hline Constant impedance & 1288.42 \\
(I) & 217.60 \\
(II) & 213.50 \\
(III) & 200.89 \\
\hline \hline
\end{tabular}

Table 5. Cost function comparison for $M_{b}=0.1$ for several incident SPL, $f=1528 \mathrm{~Hz}$

\begin{tabular}{ccc}
\hline \hline Case & \multicolumn{2}{c}{ Cost function } \\
& Formulation (0) & Formulation (I) \\
\hline \#1 Multi sine 120dB incident SPL per tone & 8.43 & 7.45 \\
\#2 Multi sine 130dB incident SPL per tone & 49.25 & 27.71 \\
\#3 Mono sine 140dB incident SPL & 362.21 & 154.17 \\
\#4 Mono sine 150dB incident SPL & 1288.42 & 217.60 \\
\hline \hline
\end{tabular}




\section{Analysis of the educed results based on formulation (I)}

For this part, only the four cases with the same Mach number (\#1 to \#4) are studied.

The numerical values of the relevant educed parameters for the affine condition $\left(R_{L}, D\right.$ and $\left.L_{\tau} / L_{\text {total }}\right)$ are compared with the classical resistance $R$ in Table 6. For the three cases with a high incident SPL (over $130 \mathrm{~dB}$ per tone), the values of $D$ are very close. For case \#1, $D$ has a lower value, which suggests that the effect is slightly different in this case. The values of $R_{L}$ are very close for all four cases, and the ratio $L_{\tau} / L_{\text {Total }}$ increases with the incident SPL. These results indicate that the affine boundary condition is able to take into account the effects of an increase of the incident SPL. The other educed values (the reactance and the incident source characteristics) remain close to each other even when the variable impedance formulations are used, as seen in Tables 7 and 8 . The reactance values with formulation (I) are always lower than the values with formulation $(0)$, however the difference is constant, small $(<0.05)$, and independent from the SPL. This highlights the consistency of the new boundary condition model, as only the educed parameters relative to the impedance condition are modified.

Case \#1 seems to be a limit case where the affine condition is not optimal (as the gain in cost function is small), even if the educed values of $R_{L}$ and $L_{\tau} / L_{\text {total }}$ are coherent with the other cases. More details on the limitations are presented in Part $\mathrm{C}$.

Figure 8 illustrates the eduction results. For each incident SPL level, the new liner impedance condition (solid lines) and the constant impedance condition (dashed lines) are plotted. The three profiles are very similar, and the coherence of the new condition is clear: the resistance value at $x=0$ increases with the incident SPL, but the values at the end of the affine evolution are very close for all three cases.

Figure 9 shows the evolution of the liner resistance value along the streamwise direction for the two monosine cases with OASPL $140 \mathrm{~dB}$ and $150 \mathrm{~dB}$ (\#3 and \#4). The curve is superimposed with the sound pressure field obtained by a LEE simulation with the educed impedance values imposed at the lined wall boundary. For high SPL on the liner, the resistance is high and decreases with the SPL. As soon as a threshold value of the SPL is achieved, the educed resistance is constant along the liner. The threshold value is around $130 \mathrm{~dB}$ for both cases. This behavior shows that with the affine impedance condition, the non-linear effects directly linked to the high SPL are visible. It is also interesting to note that on Fig. 7. the points when the decrease of the resistance stops are close for the two polynomial models. This indicates that the threshold is indeed linked to the physical effect regardless of the chosen formulation of the impedance model.

Table 6. Resistance value for $M_{b}=0.1$ for the constant (0) and the affine (I) boundary conditions, $f=1528 \mathrm{~Hz}$

\begin{tabular}{lllll}
\hline \hline \multicolumn{1}{c}{ Case } & $R$ & $R_{L}$ & $D$ & $L_{\tau} / L_{\text {total }}$ \\
\hline \#1 Multi sine 120dB incident SPL per tone & 0.211 & 0.093 & -5.95 & 0.41 \\
\#2 Multi sine 130dB incident SPL per tone & 0.307 & 0.114 & -7.41 & 0.47 \\
\#3 Mono sine 140dB incident SPL & 0.345 & 0.112 & -7.16 & 0.53 \\
\#4 Mono sine 150dB incident SPL & 0.52 & 0.14 & -7.64 & 0.69 \\
\hline \hline
\end{tabular}

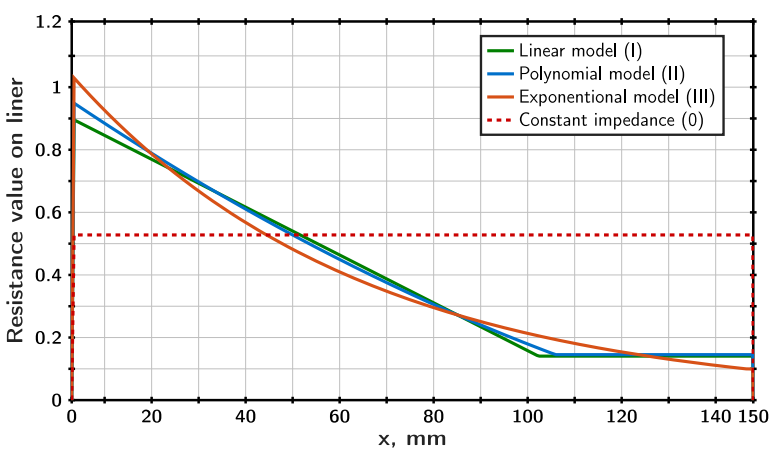

Figure 7. Resistance eduction for a SPL of $150 \mathrm{~dB}$ for a single tone excitation $f=1528 \mathrm{~Hz}$, comparison between the different boundary formulations

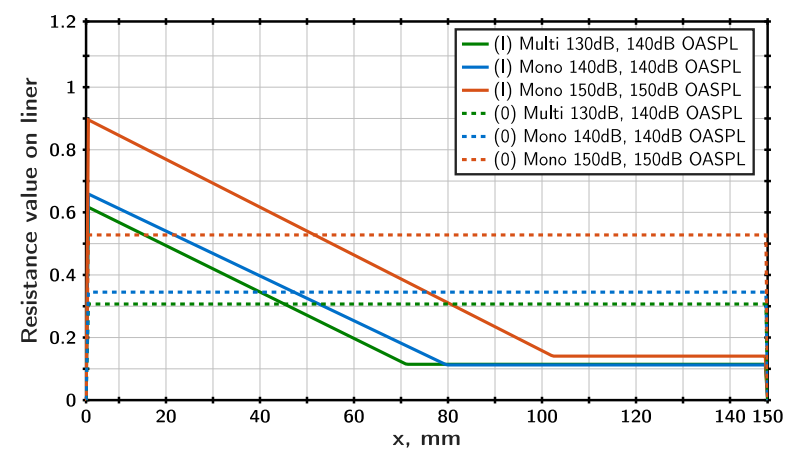

Figure 8. Resistance eduction for a single tone excitation $f=1528 \mathrm{~Hz}$, comparison between (0) and (I) boundary conditions for different incident SPLs 

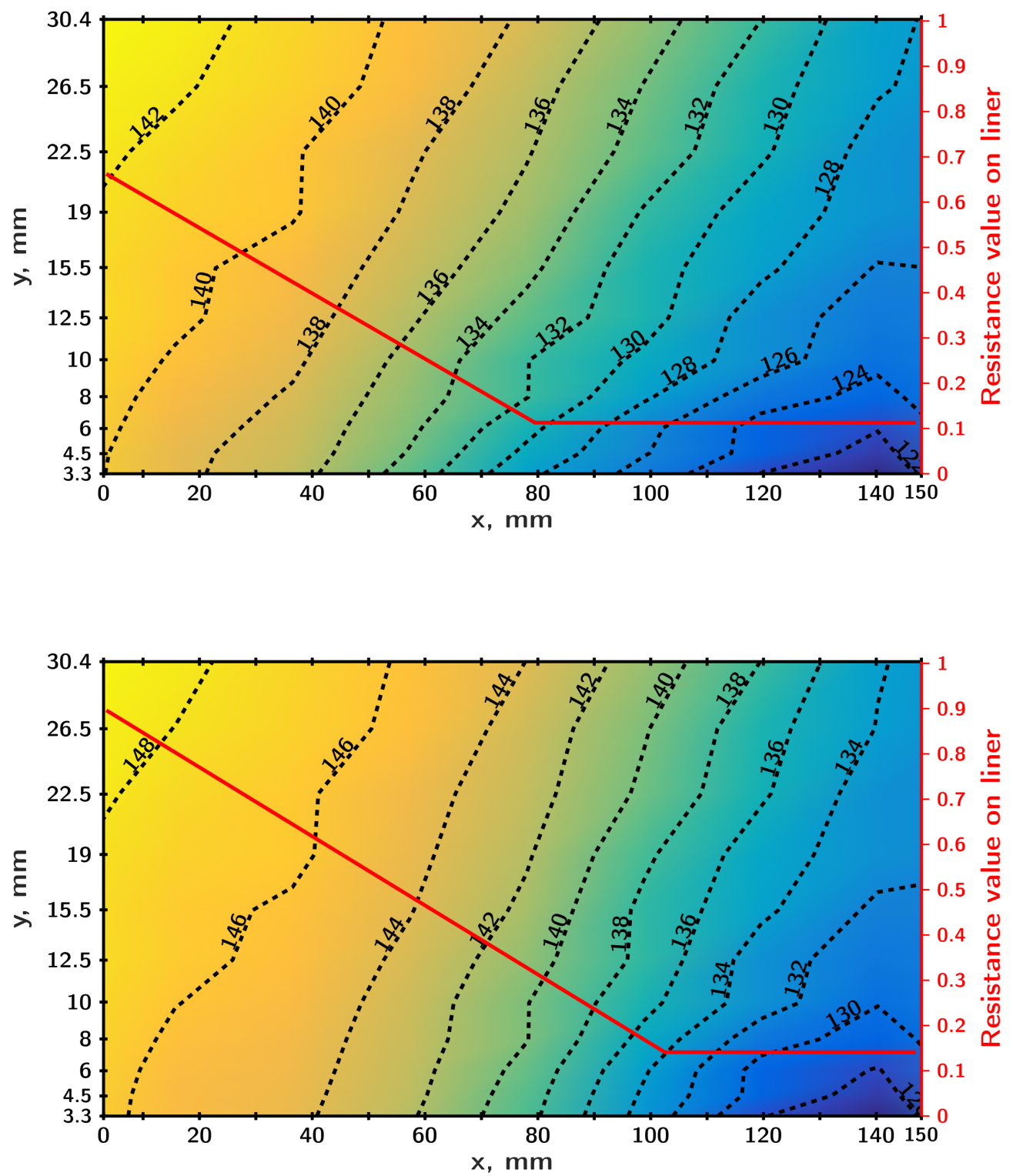

Figure 9. Resistance evolution along the liner (in red) and sound pressure field simulated with the LEE (background color and isocontours) for $M_{b}=0.1$, single tone excitation, $f=1528 \mathrm{~Hz}, S P L=140 \mathrm{~dB}$ (top) and $S P L=150 d B$ (bottom) 
Table 7. Incident source characteristics for both impedance boundary conditions

\begin{tabular}{|c|c|c|}
\hline \multirow[t]{2}{*}{ Case } & \multicolumn{2}{|c|}{ Amplitude $|\mathcal{C}|$} \\
\hline & Formulation $(0)$ & Formulation (I) \\
\hline \#1 Multi sine $120 \mathrm{~dB}$ incident SPL per tone & 0.089 & 0.090 \\
\hline \#2 Multi sine $130 \mathrm{~dB}$ incident SPL per tone & 0.333 & 0.331 \\
\hline \#3 Mono sine $140 \mathrm{~dB}$ incident SPL & 1.027 & 1.016 \\
\hline \multirow[t]{3}{*}{ \#4 Mono sine $150 \mathrm{~dB}$ incident SPL } & 2.241 & 2.193 \\
\hline & \multicolumn{2}{|c|}{ Phase $\theta_{c}$ (in degrees) } \\
\hline & Formulation $(0)$ & Formulation (I) \\
\hline \#1 Multi sine $120 \mathrm{~dB}$ incident SPL per tone & -118 & -120 \\
\hline \#2 Multi sine $130 \mathrm{~dB}$ incident SPL per tone & -126 & -127 \\
\hline \#3 Mono sine 140dB incident SPL & -119 & -120 \\
\hline \#4 Mono sine $150 \mathrm{~dB}$ incident SPL & -131 & -132 \\
\hline
\end{tabular}

Table 8. Reactance values on liner for both impedance boundary conditions

\begin{tabular}{ccc}
\hline \hline & \multicolumn{2}{c}{ Reactance } \\
& Formulation (0) & Formulation (I) \\
\hline \#1 Multi sine 120dB incident SPL per tone & 0.18 & 0.13 \\
\#2 Multi sine 130dB incident SPL per tone & 0.17 & 0.11 \\
\#3 Mono sine 140dB incident SPL & 0.17 & 0.12 \\
$\#$ \# Mono sine 150dB incident SPL & 0.13 & 0.08 \\
\hline \hline
\end{tabular}

\section{Limitations of the approach}

In this section, in order to ensure the relevance of the impedance model in other cases, the affine boundary condition was tested with the ONERA liner sample. Previous studies ${ }^{13}$ showed that this liner has a linear behavior regarding the incident SPL up to $130 \mathrm{~dB}$ : the affine impedance boundary condition is therefore expected to be unnecessary in this range. However, it should give a result coherent with the one given by the constant boundary condition.

Only the two cases with incident SPL higher than $130 \mathrm{~dB}$ were included in the eduction process. Table 9 sums up the educed results for the two considered cases (\#7 and \#8) and for both boundary conditions. For both cases, the values of $R$ and $R_{L}$ are very close. Comparing the cost functions shows that the gain obtained by changing the boundary condition is very small: for the multi-sine case, the cost function goes from 44 to 36 , and it stays exactly the same for the mono-sine case.

Figure 10 shows the educed resistances with the constant boundary condition (0) and the affine boundary condition (I) for case \#7. The constant educed resistance for case \#8 is also plotted (dashed line) for comparison. The error margins computed using the statistical method developed by Roncen et al! 7 are also added for the two constant cases (the inference method is not implemented with the affine boundary condition). One can notice that the educed values are close to each other, and that the variations all stay within the uncertainty margin. This means that there are no effects of the incident SPL on the resistance of the ONERA sample for this bulk Mach number condition.

Table 9. Resistance value for the ONERA sample, for $M_{b}=0.2$ for both liner impedance boundary conditions, $f=1992 \mathbf{H z}$

\begin{tabular}{lcccc}
\hline \hline \multicolumn{1}{c}{ Case } & $R$ & $R_{L}$ & $D$ & $L_{\tau} / L_{\text {total }}$ \\
\hline \#7 Multi sine 130dB incident SPL per tone & 1.432 & 1.259 & -83.73 & 0.15 \\
\#8 Mono sine 130dB incident SPL & 1.345 & 1.345 & 37.2 & 0.007 \\
\hline \hline
\end{tabular}


For the mono-sine case \#8,D is positive and the ratio $L_{\tau} / L_{\text {total }}$ is inferior to $1 \%$. These values have little meaning compared to all the other results. However, as the resistance values and cost functions are exactly the same, this result confirms the consistency of our affine boundary condition: it is capable of giving back the constant impedance as a result when a variable impedance is not needed (in this case, due to the linear behavior of the sample). It also indicates that the variable impedance profile that was found in case \#7 is questionable.

It should also be noted that the effect of the grazing flow is more significant than the effect of the SPL. Thus, at some point, the effect of the incident SPL may be present but too low compared to the flow to be correctly taken into account, which could explain the slight difference between case \#1 and the other cases with the IFAR sample. This conflict between the two effects also makes it difficult to conclude on the results given by the variable boundary conditions without conducting a preliminary study on the behavior of the sample (using the classical constant conditions).

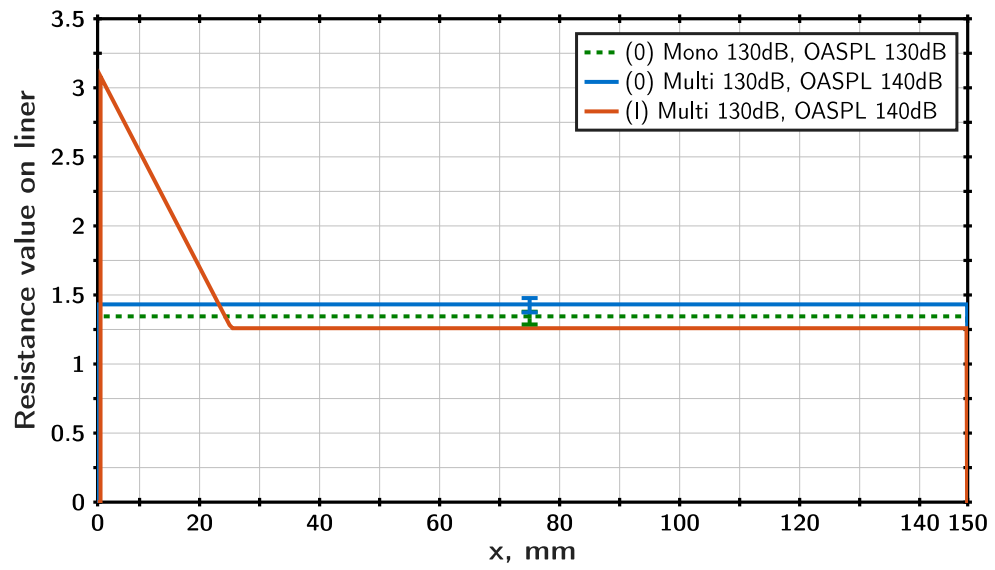

Figure 10. Resistance eduction for the two multi-sine cases on the ONERA liner sample, for both boundary conditions

\section{Conclusion}

In this paper, a new impedance eduction technique has been presented. The direct problem of wave propagation in a flow duct subject to a grazing flow was represented by the two-dimensional Linearized Euler Equations and it was solved using a Discontinuous Galerkin scheme. The classical impedance boundary condition in the eduction process was modified to include a space-dependent variable term, based on the research by Cummings. ${ }^{11}$ This modification has allowed to explain some aspects of the influence of incident SPL on the impedance of a liner. For liners with a non-linear behavior with respect to SPL, the new method has shown the existence of a threshold value above which the effects of the incident SPL on the resistance of the liner can be described with a space-dependent resistance. Under this threshold, the resistance becomes constant. The existence of this threshold confirms that SPL effects are well taken into account by using the affine condition. On the other hand, when the effect of the SPL is weaker than the grazing flow effect, a close match between the classical method and the new approach was observed, with no gain in efficiency. The new condition should thus not be used in these cases, even if its agreement with previous results is quite good. The use of this affine formulation should be useful in comparing similar samples tested in different setups. Indeed, as the educed resistance is fully independent from the sample length, different setups using different sample length are expected to find the same results for a given liner.

\section{References}

\footnotetext{
${ }^{1}$ Jing, X., Peng, S., and Sun, X., "A straightforward method for wall impedance eduction in a flow duct," The Journal of the Acoustical Society of America, Vol. 124, No. 1, 2008, pp. 227-234. doi 10.1121/1.2932256.

${ }^{2}$ Elnady, T., Bodén, H., and Elhadidi, B., "Validation of an Inverse Semi-Analytical Technique to Educe Liner Impedance," AIAA Journal, Vol. 47, No. 12, 2009, pp. 2836-2844. doi 10.2514/1.41647.

${ }^{3}$ Primus, J., Simon, F., and Piot, E., "Validation of a direct propagation model for liner impedance eduction," Proceedings
} 
of the 17th AIAA/CEAS Aeroacoustics Conference, AIAA-2011-2869, Portland, Oregon, June 2011. doi 10.2514/6.2011-2869

${ }^{4}$ Primus, J., Piot, E., and Simon, F., "Evaluation of an adjoint-based liner impedance eduction technique," Proceedings of the Acoustics 2012 Nantes Conference, Nantes, France, 23-27 April 2012.

${ }^{5}$ Primus, J., Piot, E., and Simon, F., "An adjoint-based method for liner impedance eduction: Validation and numerical investigation," Journal of Sound and Vibration, Vol. 332, No. 1, 2013, pp. 58 - 75. doi 10.1016/j.jsv.2012.07.051

${ }^{6}$ Zhou, L. and Bodén, H., "A systematic uncertainty analysis for liner impedance eduction technology," Journal of Sound and Vibration, Vol. 356, 2015, pp. 86 - 99. doi 10.1016/j.jsv.2015.07.001

${ }^{7}$ Roncen, R., Méry, F., Piot, E., and Simon, F., "Statistical Inference Method for Liner Impedance Eduction with a Shear Grazing Flow," AIAA Journal, Vol. 57, No. 3, Dec 2018, pp. 1055-1065. doi 10.2514/1.J057559

${ }^{8}$ Hersh, A. S. and Walker, B., "The Acoustic Behavior of Helmholtz Resonators Exposed to High Speed Grazing Flows," AIAA 3rd AeroAcoustics Conference, July 1976.

${ }^{9}$ Zhang and Boden, "Numerical investigation of a honeycomb liner grazed by laminar and turbulent boundary layers," $J F M$, Vol. 792, April 2016, pp. 936-980. doi $10.1017 /$ jfm.2016.79

${ }^{10}$ Rienstra, S. W. and Singh, D. K., "Nonlinear asymptotic impedance model for a Helmholtz resonator of finite depth," AIAA Journal, Vol. 56, No. 5, 2018, pp. 1792-1802. doi $10.2514 / 1 . J 055882$

${ }^{11}$ Cummings, A., "The response of a resonator under a turbulent boundary layer to a high amplitude non-harmonic sound field," Journal of Sound and Vibration, Vol. 115, No. 2, 1987, pp. 321 - 328. doi 10.1016/0022-460X(87)90475-5

12 Jones, M. G., Watson, W. R., Nark, D. M., and Howerton, B. M., "Impedance Eduction for Multisegment Liners," 2018 AIAA/CEAS Aeroacoustics Conference, 2018, p. 3441. doi 10.2514/6.2018-3441

${ }^{13}$ Primus, J., Piot, E., Simon, F., Jones, M. G., and Watson, W., "ONERA-NASA cooperative effort on liner impedance eduction," 19th AIAA/CEAS Aeroacoustics Conference, American Institute of Aeronautics and Astronautics, May 2013, p. 2273. doi $10.2514 / 6.2013-2273$

${ }^{14}$ Malmary, C., Etude Théorique et Expérimentale de L'impédance Acoustique de Matériaux En Présence D'un Ecoulement D'air Tangentiel, Ph.D. thesis, Université du Maine, 2000.

${ }^{15}$ Minotti, A., Contribution à la mesure aéroacoustique par Vélocimétrie Laser Doppler, Ph.D. thesis, Toulouse, ENSAE, 2004.

${ }^{16}$ Minotti, A., Simon, F., and Gantié, F., "Characterization of an acoustic liner by means of Laser Doppler Velocimetry in a subsonic flow," Aerospace Science and Technology, Vol. 12, No. 5, 2008, pp. 398 - 407. doi 10.1016/j.ast.2007.09.007.

${ }^{17}$ Chung, J. Y. and Blaser, D. A., "Transfer function method of measuring in-duct acoustic properties. I. Theory," The Journal of the Acoustical Society of America, Vol. 68, No. 3, 1980, pp. 907-913. doi 10.1121/1.384778.

${ }^{18}$ Bodén, H. and Åbom, M., "Influence of errors on the two-microphone method for measuring acoustic properties in ducts," The Journal of the Acoustical Society of America, Vol. 79, No. 2, 1986, pp. 541-549. doi 10.1121/1.393542

${ }^{19}$ Mery, F., Sebbane, D., Piot, E., Simon, F., Reulet, P., and Carazo-Mendez, A., "Experimental assessment of the effect of temperature gradient across an aeroacoustic liner," 2018 AIAA/CEAS Aeroacoustics Conference, 2018, p. 4100. doi $10.2514 / 6.2018-4100$

${ }^{20}$ Leon, O., Piot, E., Sebbane, D., and Simon, F., "Measurement of acoustic velocity components in a turbulent flow using LDV and high-repetition-rate PIV," Experiments in fluids, 2017. doi 10.1007/s00348-017-2348-4

${ }^{21}$ Nance, D. and Ahuja, K. K., "Limitations of the three-microphone signal enhancement technique," Proceedings of the 45th AIAA Aerospace Sciences Conference, AIAA-2007-441, Reno, Nevada, January 2007. doi 10.2514/6.2007-441.

${ }^{22}$ Lavieille, M., Simon, F., and Micheli, F., "Measurement of acoustic quantity fields in duct flow by Laser Doppler Velocimetry," Proceedings of the 12th AIAA/CEAS Aeroacoustics Conference, AIAA-2006-2550, Cambridge, Massachusetts, May 8-10 2006. doi 10.2514/6.2006-2550

${ }^{23}$ Pascal, L., Piot, E., and Casalis, G., "Discontinuous Galerkin Method for acoustic modes computation in lined ducts," Proceedings of the 18th AIAA/CEAS Aeroacoustics conference, AIAA 2012-2153, Colorado Springs, Colorado, USA, 4-6 June 2012. doi $10.2514 / 6.2012-2153$

${ }^{24}$ Piot, E., Primus, J., and Simon, F., "Liner impedance eduction technique based on velocity fields," Proceedings of the 18 th AIAA/CEAS Aeroacoustics Conference, AIAA 2012-2198, Colorado Springs, Colorado, USA, 4-6 June 2012. doi 10.2514/6.20122198

${ }^{25}$ Tonon, D., Moers, E., and Hirschberg, A., "Quasi-steady acoustic response of wall perforations subject to a grazing-bias flow combination," Journal of Sound and Vibration, Vol. 332, No. 7, 2013, pp. 1654-1673. doi 10.1016/j.jsv.2012.11.024 